\title{
KINETICS OF ELECTROMAGNETIC FIELD IN NONEQUILIBRIUM
} MEDIUM

\author{
S.F. Lyagushyn ${ }^{1 *}$, A.I. Sokolovsky ${ }^{1}$, S.A. Sokolovsky ${ }^{2}$ \\ ${ }^{I}$ Oles Honchar Dnipro National University, Dnipro, Ukraine \\ ${ }^{2}$ Prydniprovska State Academy of Civil Engineering and Architecture, Dnipro, Ukraine \\ *e-mail: 1yagush.new@gmail.com
}

\begin{abstract}
Kinetics of electromagnetic field in noneqilibrim medium of atoms is studied on the basis of the Dicke model. The field is described by average electric and magnetic fields and their binary correlations. The atoms are considered in two-level approximation and assumed to be fixed in the space. States of the medium are described by the energy density. Nonresonant atom-field interaction is accounted phenomenologically. The theory is built on the Bogolyubov idea of the functional hypothesis that is the basis of his method of the reduced description of nonequilibrium processes. The investigation of the system is carried out in the framework of the Peletminsky-Yatsenko model. Atom-field interaction is assumed to be small and is taken into account up to the second contributions in the interaction included. Average electric and magnetic fields satisfy the Maxwell equations. Material equations for them express average current and charge densities through the average field. Evolution equations for the binary correlation of the field are constructed. Material equations for these equations express correlations field-current trough correlations field-field. The time equation for energy density of the medium is obtained. Density evolution is governed by the average field and field correlations. All material coefficients of the system are found.

Keywords: electromagnetic field; two-level atoms; Dicke model; reduced description; PeletminskyYatsenko model; current; material equations; correlations of the field.
\end{abstract}

Received 08.11.2019; Received in revised form 17.12.2019; Accepted 23.12.2019

\section{Introduction}

Electromagnetic field kinetics is studied in the medium of atoms on the basis of the Dicke model [1] (see also [2]). Nonequilibrium states of the system are investigated by the Bogolyubov method of the reduced description based on his idea of the functional hypothesis [3] (see a review of the method in [4]). The field-atoms interaction is assumed to be small. Average magnetic, transversal electric fields, their binary correlations, and energy of the medium density are chosen as reduced description parameters. Using correlations as such parameters is necessary because statistical operator of the field described only by average quantities does not exist. Atoms of the medium are assumed to be two-level ones, nonmoving and arbitrarily located in the space. The listed above reduced description parameters allows investigating nonequilibrium states of the system in the framework of the Peletminsky-Yatsenko model [4] that simplifies our consideration.

The sketch of such a theory is presented in the conference paper [5]. The detailed analysis of the theory basics and discussion of material equations for the Maxwell equations with step by step calculations is given in [6].

The article has the following structure. Section 2 discusses the basic equations of the theory and material equations to the Maxwell equations with some substantial remarks to our paper [6]. The equation of the medium dynamics in the electromagnetic field is obtained in the Section 3. Section 4 is devoted to dynamics of binary correlations of the electromagnetic field in the nonequilibrium atomic medium and material equations to evolution equations for the correlations. Some useful relations are given in the Appendix.

\section{Basic equations of the theory and material equations for the Maxwell equations}

The kinetics of the electromagnetic field in the medium of atoms, which are considered in two-level approximation, is investigated. Atoms are fixed in space and arbitrarily distributed with the density $n(x)$. Basic equations of the theory are presented in our paper [6]. Here additional details that simplify the consideration are given. 
In the excited state, atoms of the medium have energy $\mathrm{h} \omega$ and an electric dipole moment $d_{n}$. In the generalized quasispin Dike model the Hamilton operator of the system can be written in the form [1]

$$
\begin{gathered}
\hat{H}=\hat{H}_{0}+\hat{H}_{1}, \quad \hat{H}_{0}=\hat{H}_{\mathrm{f}}+\hat{H}_{\mathrm{m}}, \quad \hat{H}_{\mathrm{f}}=\sum_{k, \alpha} \hbar \omega_{k} c_{\alpha k}^{+} c_{\alpha k}, \quad \hat{H}_{\mathrm{m}}=\hbar \omega \sum_{1 \leq a \leq N} \hat{r}_{a z}, \\
\hat{H}_{1}=-\int d x \hat{E}_{n}^{t}(x) \hat{P}_{n}(x), \quad \hat{P}_{n}(x) \equiv \sum_{a} 2 \hat{r}_{a x} d_{a n} \delta\left(x-x_{a}\right)
\end{gathered}
$$

(see discussion in the paper [2]). Here $\hat{H}_{\mathrm{f}}$ is the Hamiltonian of the free electromagnetic field ( $\left.\omega_{k} \equiv c k\right), \hat{r}_{a n}$ and $d_{a n}$ are the quasispin operator and the dipole moment of the $a$-th atom. The vectors $d_{a n}$ of different atoms differ only in their orientation $\left(d_{a n} d_{a n} \equiv d^{2}\right)$, which is described by the distribution function $w_{d} . \hat{P}_{n}(x)$ is the operator of electric dipole moment density of the system (in other word, polarization) [2]. The interaction of atoms and the field is described by the operator $\hat{H}_{1}$ with the transverse electric field $\hat{E}_{n}^{t}(x)$ (the dipole-dipole interaction of atoms is neglected). Here and further periodic boundary conditions and other standard notations of quantum electrodynamics are used (see, for example, [4] and (A.1), (A.2) in the Appendix). The interaction of atoms and field is weak that allows considering the dipole moment $d$ of an atom as a formal small parameter.

The nonequilibrium states of the field will be described by parameters $\eta_{a}: \zeta_{\mu}$, $\left(\zeta_{\mu}, \zeta_{\mu^{\prime}}\right)$ where $\zeta_{\mu}$ are average transverse electric $E_{n}^{t}(x)$ and magnetic $B_{n}(x)$ fields and their binary correlations $\left(\zeta_{\mu}, \zeta_{\mu^{\prime}}\right)$. Here and below the following compact notations

$$
\begin{gathered}
\zeta_{\mu}=\zeta_{i n}(x), \quad \zeta_{1 n}(x)=E_{n}^{t}(x), \quad \zeta_{2 n}(x)=B_{n}(x), \quad \zeta_{\mu}=\operatorname{Sp} \rho \hat{\zeta}_{\mu} \\
\hat{\zeta}_{\mu}=\hat{\zeta}_{i n}(x), \quad \hat{\zeta}_{1 n}(x)=\hat{E}_{n}^{t}(x), \quad \hat{\zeta}_{2 n}(x)=\hat{B}_{n}(x) \\
\sum_{\mu} \ldots=\sum_{i n} \int_{V} d x \ldots ; \quad \zeta_{s}=\zeta_{\mu_{s}}, \quad \sum_{s} \ldots=\sum_{\mu_{s}} \ldots
\end{gathered}
$$

are used (the corresponding operators $\hat{E}_{n}^{t}(x)$ and $\hat{B}_{n}(x)$ are given in (A.3), (A.4)). Further the average value $a$ of a quantity is denoted by the same letter as its operator $\hat{a}$.

The general definition of the binary correlation function of operators $\hat{a}$ and $\hat{b}$ is given by the formula with the statistical operator $\rho$ of the system and their anticommutator

$$
(a, b)=\operatorname{Sp} \rho\{\hat{a}, \hat{b}\} / 2-\operatorname{Sp} \rho \hat{a} \operatorname{Sp} \rho \hat{b} .
$$

Note, the necessity of taking into account at least binary correlations of the field in describing its state is related to the nonexistence of a statistical operator that describes the field only by its average quantities $E_{n}^{t}(x), B_{n}(x)$.

The state of the medium in this paper is described by the average density $\varepsilon(x)$ of its energy 


$$
\varepsilon(x)=\operatorname{Sp} \rho \hat{\varepsilon}(x), \quad \hat{\varepsilon}(x)=\sum_{a} \hbar \omega \hat{r}_{a z} \delta\left(x-x_{a}\right)
$$

and assumed to be a local equilibrium one.

Thus, the values $\eta_{a}$ and $\varepsilon(x)$ are the reduced description parameters for states of the system. Statistical operator at the reduced description $\rho(\eta, \varepsilon)$ is a functional of variables $\eta_{a}: \zeta_{\mu},\left(\zeta_{\mu}, \zeta_{\mu^{\prime}}\right)$ and $\varepsilon(x)$. The state is observed at times $t \gg \tau_{0}$ where some time $\tau_{0}$ depends on the initial state of the system. For the statistical operator $\rho(\eta, \varepsilon)$ formulas

$$
\operatorname{Sp} \rho(\eta, \varepsilon) \hat{\varepsilon}=\varepsilon, \quad \operatorname{Sp} \rho(\eta, \varepsilon) \hat{\eta}_{a}=\eta_{a}
$$

are exact.

When constructing system evolution equations, it is convenient to use the operator form of the evolution equations $\hat{\dot{a}} \equiv i[\hat{H}, \hat{a}] / \hbar$. Really, for an arbitrary operator $\hat{a}$ the quantum Liouville equation for a statistical operator $\rho(t)$ gives an evolution equation

$$
\partial_{t} \operatorname{Sp} \rho(t) \hat{a}=\operatorname{Sp} \rho(t) \hat{\dot{a}} .
$$

Here $\hat{\dot{a}}$ is the velocity change operator for the quantity described with an operator $\hat{a}$ (sometimes it is convenient to apply the notation $\hat{\dot{a}} \equiv \partial_{t} \hat{a}$, although all operators in this paper are taken in the Schrödinger picture). Maxwell's equations and evolution equation for the medium energy density in the operator form take the expected view

$$
\hat{\dot{B}}_{n}=-c \operatorname{rot}_{n} \hat{E}, \quad \hat{\dot{E}}_{n}=c \operatorname{rot}_{n} \hat{B}-4 \pi \hat{I}_{n}, \quad \operatorname{div} \hat{B}=0, \quad \operatorname{div} \hat{E}=4 \pi \hat{\rho} ; \quad \hat{\dot{\varepsilon}}=\hat{I}_{n} \hat{E}_{n}^{t} .
$$

Here the operators of the complete electric field $\hat{E}_{n}$, densities of current $\hat{I}_{n}$ and charge $\hat{\rho}$ of the system are defined by the formulas

$$
\hat{E}_{n}=\hat{E}_{n}^{t}-4 \pi \hat{P}_{n}, \quad \hat{I}_{n}=\hat{\dot{P}}_{n}, \quad \hat{\rho}=-\operatorname{div} \hat{P}, \quad \hat{I}_{n}=-\sum_{a} 2 \omega d_{a n} \hat{r}_{a y} \delta\left(x-x_{a}\right) .
$$

Relations (7) can easily be proved taking into account formulas (A.3), (A.4) from the Appendix. The second formula in (8) is natural because atoms of the medium do not move and have no magnetic dipole moment. The first formula in (8) for the operator of the total electric field $\hat{E}_{n}$ leads to first two Maxwell equations in (7).

In our paper [6] it is shown that nonequilibrium states of the considered system can be described by the reduced description parameters $\eta_{a}$ and $\varepsilon$ in the framework of the Peletminsky-Yatsenko model [4]. In this model, the statistical operator $\rho(\eta, \varepsilon)$ is calculated in perturbation theory in interaction $\hat{H}_{1}$ from an integral equation obtained for the considered problem in [4]. Statistical operator of the system $\rho(\eta, \varepsilon)$ has the structure

$$
\rho(\eta, \varepsilon)=\rho^{(0)}+\rho^{(1)}+O\left(d^{2}\right), \quad \rho^{(0)}=\rho_{q}(\eta, \varepsilon)
$$

$\left(a^{(s)}\right.$ is the contribution of the order $d^{s}$ to the value $a$ ). The statistical operator $\rho_{q}(\eta, \varepsilon)$ is called the quasi-equilibrium one and according to the Peletminsky-Yatsenko model, looks like [6] 


$$
\begin{gathered}
\rho_{q}(\eta, \varepsilon)=\rho_{\mathrm{f}}(\eta) \rho_{\mathrm{m}}(\varepsilon), \quad \rho_{\mathrm{m}}(\varepsilon)=w_{\mathrm{r}}(\varepsilon) w_{\mathrm{d}} w_{\alpha} ; \\
\rho_{\mathrm{f}}(\eta)=\exp \left\{\Omega(\eta)-\sum_{a} Z_{a}(\eta) \hat{\eta}_{a}\right\}, \quad \operatorname{Sp}_{\mathrm{f}} \rho_{\mathrm{f}}(\eta)=1, \quad \operatorname{Sp}_{\mathrm{f}} \rho_{\mathrm{f}}(\eta) \hat{\eta}_{a}=\eta_{a} ; \\
w_{\mathrm{r}}(\varepsilon)=\exp \left\{\Phi(\varepsilon)-\int d x Z(x, \varepsilon) \hat{\varepsilon}(x)\right\}, \quad \mathrm{Sp}_{\mathrm{r}} w_{\mathrm{r}}(\varepsilon)=1, \quad \operatorname{Sp}_{\mathrm{r}} w_{\mathrm{r}}(\varepsilon) \hat{\varepsilon}(x)=\varepsilon(x) ; \\
w_{\alpha}(\omega)=\frac{\alpha c(\alpha)}{\left(\omega-\omega_{0}\right)^{2}+\alpha^{2}} \quad\left(\alpha<<\omega_{0}\right), \quad \int_{0}^{+\infty} d \omega w_{\alpha}(\omega)=1 ; \quad \operatorname{Sp}_{\mathrm{d}} w_{\mathrm{d}}=1 ; \\
\operatorname{Sp}_{\ldots}=\operatorname{Sp}_{\mathrm{f}} \operatorname{Sp}_{\mathrm{m}} \ldots, \quad \mathrm{Sp}_{\mathrm{m}} \ldots=\operatorname{Sp}_{\alpha} \operatorname{Sp}_{\mathrm{r}} \operatorname{Sp}_{\mathrm{d}} \ldots .
\end{gathered}
$$

Here $\rho_{\mathrm{f}}(\eta)$ is a quasi-equilibrium statistical operator of the electromagnetic field. The operator $\hat{\eta}_{a}$ in $\rho_{\mathrm{f}}(\eta)$ includes field operators $\hat{E}_{n}^{t}, \hat{B}_{n}$ and all their anticommutators (the last term in (3) is numerical and corrects only $\Omega(\eta)$ ). Therefore, in the exponent $\rho_{\mathrm{f}}(\eta)$ there is a quadratic form of Bose operators of the field, which ensures the existence of traces with it. The operator $w_{\mathrm{r}}(\varepsilon)$ is a locally equilibrium statistical operator of the medium and therefore $T(x, \varepsilon)=Z(x, \varepsilon)^{-1}$ is the inverse temperature of the medium. Traces with the operator $w_{\mathrm{r}}(\varepsilon)$ are taken in the quasispin space (see (A.7), (A.8) in the Appendix). Here additional to [6] function $w_{\alpha}$ is added to $\rho_{\mathrm{m}}(\varepsilon)$ in order to take into account nonresonant interaction of the atoms with the electromagnetic field phenomenologically ( $\alpha$ is the width of the energy level of an atom with energy h $\omega_{0}$; $\left.\lim _{\alpha \rightarrow 0} w_{\alpha}=\delta\left(\omega-\omega_{0}\right)\right)$. Value $w_{\mathrm{d}}$ is the distribution of the orientations of the dipoles of the atoms of the medium. Hereafter (as in [6]), for simplicity it is assumed that the distribution of dipole moments of atoms is isotropic one and there are no correlations between them. The last is natural, because here atom-atom interaction is neglected.

In the paper [6], it is shown that the first order contribution to the statistical operator $\rho(\varepsilon, \eta)$ is given by

$$
\rho^{(1)}=-\frac{i}{\hbar} \int_{-\infty}^{0} d \tau \int d x\left[\rho_{\mathrm{f}} \rho_{\mathrm{m}}, \hat{E}_{n}^{t}(x, \tau) \hat{P}_{n}(x, \tau)\right]
$$

where operators in the Dirac picture

$$
\hat{E}_{n}^{t}(x, \tau) \equiv e^{\frac{i}{\hbar} \tau \hat{H}_{\mathrm{f}}} \hat{E}_{n}^{t}(x) e^{-\frac{i}{\hbar} \tau \hat{H}_{\mathrm{f}}}, \quad \hat{P}_{n}(x, \tau) \equiv e^{\frac{i}{\hbar} \tau \hat{H}_{\mathrm{m}}} \hat{P}_{n}(x) e^{-\frac{i}{\hbar} \tau \hat{H}_{\mathrm{m}}}
$$

are taken.

As a result, average values of polarization

$$
\begin{aligned}
& P_{n}(x)=\int_{V} d x^{\prime}\left[k\left(x-x^{\prime}, \varepsilon(x)\right) E_{n}^{t}\left(x^{\prime}\right)+c \lambda\left(x-x^{\prime}, \varepsilon(x)\right) Z_{n}\left(x^{\prime}\right)\right]+O\left(d^{3}\right), \\
& \kappa(k, \varepsilon)=-\varepsilon \frac{4 d^{2}}{3 \hbar^{2}} \frac{1}{\omega_{0}^{2}-\omega_{k}^{2}} \quad(\alpha \rightarrow 0), \quad \lambda(k, \varepsilon)=\frac{2 \pi d^{2}}{3 \hbar^{2}} \varepsilon \frac{w_{\alpha}\left(\omega_{k}\right)}{\omega_{k}^{2}} ; \\
& Z_{n} \equiv \operatorname{rot}_{n} B
\end{aligned}
$$


and current density

$$
\begin{gathered}
I_{n}(x)=\int_{V} d x^{\prime}\left[\sigma\left(x-x^{\prime}, \varepsilon(x)\right) E_{n}^{t}\left(x^{\prime}\right)+c \xi\left(x-x^{\prime}, \varepsilon(x)\right) Z_{n}\left(x^{\prime}\right)\right]+O\left(d^{3}\right), \\
\sigma(k, \varepsilon) \equiv-\lambda(k, \varepsilon) \omega_{k}^{2}, \quad \xi(k, \varepsilon) \equiv \kappa(k, \varepsilon)
\end{gathered}
$$

are obtained. Quantities $\kappa(k, \varepsilon), \lambda(k, \varepsilon), \sigma(k, \varepsilon)$, and $\xi(k, \varepsilon)$ are the Fourier transforms of the functions $\kappa(x, \varepsilon), \lambda(x, \varepsilon), \sigma(x, \varepsilon)$, and $\xi(x, \varepsilon)$ defined according to (A.5)) in the Appendix. Additional to [6], here the nonresonant interaction of the atoms and electromagnetic field is accounted phenomenologically. Relations (13) and (14) are material equations to the Maxwell equations. The material coefficient $\kappa(k, \varepsilon)$ in (13) has the sense of dielectric susceptibility. In our terms the coefficient $\lambda(k, \varepsilon)$ describes the effects of the frequency dispersion because according to (7) and (8) $Z_{n}=c^{-1} \partial_{t} E_{n}^{t}$. The material coefficients $\sigma(k, \varepsilon)$ and $\xi(k, \varepsilon)$ in (14) have the sense of conductivity and magnetic susceptibility. From the other side, in our terms the material coefficient $\xi(k, \varepsilon)$ describes the effects of the frequency dispersion.

Expressions (13) and (14) can be written with the same accuracy in the form with the total electric field $E_{n}(x)$ because material coefficients $\kappa(k, \varepsilon), \lambda(k, \varepsilon), \sigma(k, \varepsilon)$, $\xi(k, \varepsilon) \sim d^{2}$ and according to (8) $E_{n}^{t}=E_{n}+4 \pi P_{n}=E_{n}+O\left(d^{2}\right)$.

\section{Medium dynamics in the electromagnetic field}

The material equations (13) and (14) include the energy density of the medium $\varepsilon$ as a reduced description parameter. Here the evolution equation for this quantity is obtained. According to (6), (7), and (11) the expressions

$$
\begin{gathered}
\partial_{t} \varepsilon(x)=\operatorname{Sp} \rho(\eta, \varepsilon) \hat{I}_{n}(x) \hat{E}_{n}^{t}(x), \\
\operatorname{Sp}^{(1)} \hat{I}_{n}(x) \hat{E}_{n}^{t}(x)=-\frac{i}{\hbar} \int_{-\infty}^{0} d \tau \int d x^{\prime} \operatorname{Sp}_{\mathrm{f}} \rho_{\mathrm{f}} \hat{E}_{l}^{t}\left(x^{\prime}, \tau\right) \hat{E}_{n}^{t}(x) \operatorname{Sp}_{\mathrm{m}} \rho_{\mathrm{m}}\left[\hat{P}_{l}\left(x^{\prime}, \tau\right), \hat{I}_{n}(x)\right] \\
-\frac{i}{\hbar} \int_{-\infty}^{0} d \tau \int d x^{\prime} \operatorname{Sp}_{\mathrm{f}} \rho_{\mathrm{f}}\left[\hat{E}_{l}^{t}\left(x^{\prime}, \tau\right), \hat{E}_{n}^{t}(x)\right] \operatorname{Sp}_{\mathrm{m}} \rho_{\mathrm{m}} \hat{I}_{n}(x) \hat{P}_{l}\left(x^{\prime}, \tau\right) \equiv L_{1}^{(2)}(x)+L_{2}^{(2)}(x)
\end{gathered}
$$

are true (in [6] it is shown that $\left.\operatorname{Sp}^{(0)} \hat{I}_{n}(x) \hat{E}_{n}^{t}(x)=0\right)$. In (15), averaging with $\rho_{\mathrm{m}}$ in $L_{1}^{(2)}(x)$ gives for the isotropic distribution of dipole moments of atoms

$$
\operatorname{Sp}_{\mathrm{m}} \rho_{\mathrm{m}}\left[\hat{P}_{l}\left(x^{\prime}, \tau\right), \hat{I}_{n}(x)\right]=-i \frac{4 d^{2}}{3 \hbar} \varepsilon(x) \delta\left(x-x^{\prime}\right) \delta_{n l} \int_{0}^{+\infty} d \omega w_{\alpha}(\omega) \cos \omega \tau
$$

Average with $\rho_{\mathrm{f}}$ in $L_{1}^{(2)}(x)$ is transformed consistently as

$$
\operatorname{Sp}_{\mathrm{f}} \rho_{\mathrm{f}} \hat{E}_{l}^{t}\left(x^{\prime}, \tau\right) \hat{E}_{n}^{t}(x)=\frac{1}{V^{2}} \sum_{k, k^{\prime}} e^{i\left(k x+k^{\prime} x^{\prime}\right)} \operatorname{Sp}_{\mathrm{f}} \rho_{\mathrm{f}}\left(\hat{E}_{n k^{\prime}}^{t} \cos \omega_{k^{\prime}} \tau+c \hat{Z}_{n k^{\prime}} \omega_{k^{\prime}}^{-1} \sin \omega_{k^{\prime}} \tau\right) \hat{E}_{n k}^{t}=
$$




$$
\begin{gathered}
\quad=\frac{1}{V^{2}} \sum_{k, k^{\prime}} e^{i\left(k x+k^{\prime} x^{\prime}\right)}\left\{\left[\left(E_{l k^{\prime}}^{t}, E_{n k}^{t}\right)+E_{l k^{\prime}}^{t} E_{n k}^{t}\right] \cos \omega_{k^{\prime}} \tau+\right. \\
\left.+\left(\left[\left(Z_{l k^{\prime}}, E_{n k}^{t}\right)+Z_{l k^{\prime}} E_{n k}^{t}\right]+2 \pi i h c\left(k_{n} k_{l}-k^{2} \delta_{n l}\right) \delta_{k^{\prime},-k}\right) c \omega_{k^{\prime}}^{-1} \sin \omega_{k^{\prime}} \tau\right\} .
\end{gathered}
$$

According to relations (10), this formula includes average fields and binary correlations and formulas of the type

$$
E_{n}^{t}=\operatorname{Sp}_{\mathrm{f}} \rho_{\mathrm{f}} \hat{E}_{n}^{t}, \quad\left(E_{l k^{\prime}}^{t}, E_{n k}^{t}\right)=\operatorname{Sp}_{\mathrm{f}} \rho_{\mathrm{f}}\left(\frac{1}{2}\left\{\hat{E}_{l k^{\prime}}^{t}, \hat{E}_{n k}^{t}\right\}-E_{l k^{\prime}}^{t} E_{n k}^{t}\right)
$$

taken with the statistical operator $\rho_{\mathrm{f}}$ are exact. The transition from the first expression in (17) to the second one is made using identities

$$
\hat{a} \hat{b}=\frac{1}{2}\{\hat{a}, \hat{b}\}+\frac{1}{2}[\hat{a}, \hat{b}], \quad\left[\hat{Z}_{n k}, \hat{E}_{l k^{\prime}}^{t}\right]=2 \pi i \mathrm{~h} c\left(k_{n} k_{l}-k^{2} \delta_{n l}\right) \delta_{k^{\prime},-k}, \quad\left[\hat{E}_{n k}^{t}, \hat{E}_{l k^{\prime}}^{t}\right]=0
$$

(see also (A.6) in the Appendix). Taking into account (16) and (17) with (15) leads to the expression for $L_{1}^{(2)}(x)$

$$
\begin{aligned}
& L_{1}^{(2)}(x)=-\frac{4 d^{2}}{3 \hbar^{2}} \int_{-\infty}^{0} d \tau \int_{0}^{+\infty} d \omega w_{\alpha}(\omega) \frac{1}{V^{2}} \sum_{k, k^{\prime}} e^{i\left(k x+k^{\prime} x^{\prime}\right)}\left\{\left[\left(E_{n k^{\prime}}^{t}, E_{n k}^{t}\right)+E_{n k^{\prime}}^{t} E_{n k}^{t}\right] \cos \omega_{k^{\prime}} \tau+\right. \\
& \left.+\left[\left(Z_{n k^{\prime}}, E_{n k}^{t}\right)+Z_{n k^{\prime}} E_{n k}^{t}\right] c \omega_{k^{\prime}} \sin \omega_{k^{\prime}} \tau-\delta_{k^{\prime},-k} 4 \pi i \hbar \omega_{k} \sin \omega_{k} \tau\right\} \varepsilon(x) \cos \omega \tau .
\end{aligned}
$$

Calculating $L_{2}^{(2)}(x)$ for the isotropic distribution of atom dipoles gives

$$
\begin{aligned}
& \operatorname{Sp}_{\mathrm{f}} \rho_{\mathrm{f}}\left[\hat{E}_{l}^{t}\left(x^{\prime}, \tau\right), \hat{E}_{n}^{t}(x)\right]=4 \pi i \mathrm{~h} \frac{1}{V} \sum_{k} e^{i k\left(x-x^{\prime}\right)}\left(k_{n} k_{l}-k^{2} \delta_{n l}\right) \omega_{k} \sin \omega_{k} \tau \\
& \operatorname{Sp}_{\mathrm{m}} \rho_{\mathrm{m}} \hat{I}_{n}(x) \hat{P}_{l}\left(x^{\prime}, \tau\right)= \frac{4 d^{2}}{3} \delta\left(x-x^{\prime}\right) \delta_{n l} \sum_{a} \delta\left(x-x_{a}\right) \times \\
& \times \int_{0}^{+\infty} d \omega w_{\alpha}(\omega) \operatorname{Sp}_{\mathrm{r}} w_{\mathrm{r}}\left\{-\hat{r}_{a x} \hat{r}_{a y} \cos \omega \tau+\hat{r}_{a y}^{2} \omega \sin \omega \tau\right\}
\end{aligned}
$$

The Pauli matrixes representation method (see about it in (A.8) from the Appendix) allows easily proving formulas

$$
\mathrm{Sp}_{\mathrm{r}} w_{\mathrm{r}} \hat{r}_{a x} \hat{r}_{a y}=-\frac{i}{2} \mathrm{Sp}_{\mathrm{r}} w_{\mathrm{r}} \hat{r}_{a z}, \quad \mathrm{Sp}_{\mathrm{r}} w_{\mathrm{r}} \hat{r}_{a y}^{2}=\frac{1}{4}
$$

which give

$$
\operatorname{Sp}_{\mathrm{m}} \rho_{\mathrm{m}} \hat{I}_{n}(x) \hat{P}_{l}\left(x^{\prime}, \tau\right)=\frac{d^{2}}{3} \delta\left(x-x^{\prime}\right) \delta_{n l} \int_{0}^{+\infty} d \omega w_{\alpha}(\omega)[2 i \varepsilon(x) \cos \omega \tau+\omega n(x) \sin \omega \tau]
$$




$$
n(x) \equiv \sum_{a} \delta\left(x-x_{a}\right) .
$$

Here $n(x)$ is atom concentration (the density of the number of atoms), $\varepsilon(x)$ is their energy density given by (4). According to (21), (23), and (15), quantity $L_{2}^{(2)}(x)$ takes the form

$$
L_{2}^{(2)}(x)=-\frac{4 d^{2}}{3 \hbar^{2}} \int_{-\infty}^{0} d \tau \int_{0}^{+\infty} d \omega w_{\alpha}(\omega) \frac{1}{V} \sum_{k} 2 \pi \hbar \omega_{k}[i \pi \varepsilon(x) \cos \omega \tau+\hbar \omega n(x) \sin \omega \tau] \sin \omega_{k} \tau .
$$

The integrals over $\tau$ in (20) and (24) should be taken in the class of generalized functions after thermodynamic limit transition in these expressions by the usual rule

$$
\sum_{k} \ldots \stackrel{T L}{=} \frac{V}{(2 \pi)^{3}} \int d^{3} k \ldots
$$

Similarly to calculations conducted in [6], formulas

$$
\begin{aligned}
\int_{-\infty}^{0} d \tau \sin \omega_{k} \tau \sin \omega \tau= & \frac{\pi}{2} \delta\left(\omega-\omega_{k}\right), \quad \int_{-\infty}^{0} d \tau \cos \omega_{k} \tau \cos \omega \tau=\frac{\pi}{2} \delta\left(\omega-\omega_{k}\right), \\
& \int_{-\infty}^{0} d \tau \sin \omega_{k} \tau \cos \omega \tau=\mathrm{P} \frac{\omega_{k}}{\omega^{2}-\omega_{k}^{2}} .
\end{aligned}
$$

for $\omega, \omega_{k}>0$ are true. Taking into account expressions (13) and (14) for material coefficients $\sigma(k, \varepsilon)$ and $\xi(k, \varepsilon)$ gives

$$
\begin{gathered}
\operatorname{Sp}^{(1)} \hat{I}_{n}(x) \hat{E}_{n}^{t}(x)=\frac{1}{V^{2}} \sum_{k, k^{\prime}} e^{i\left(k x+k^{\prime} x^{\prime}\right)}\left\{\left[\left(E_{l k^{\prime}}^{t}, E_{n k}^{t}\right)+E_{l k^{\prime}}^{t} E_{n k}^{t}\right] \sigma\left(k^{\prime}, \varepsilon(x)\right)+\right. \\
\left.+\left[\left(Z_{l k^{\prime}}, E_{n k}^{t}\right)+Z_{l k^{\prime}} E_{n k}^{t}\right] c \xi(k, \varepsilon(x))\right\}-\frac{4 \pi d^{2}}{3} n(x) \int_{0}^{+\infty} d \omega w_{\alpha}(\omega) \omega^{2} \frac{1}{V} \sum_{k} \delta\left(\omega-\omega_{k}\right) .
\end{gathered}
$$

Here the last sum in the thermodynamic limit according to (25) equals

$$
\frac{1}{V} \sum_{k} \delta\left(\omega-\omega_{k}\right) \stackrel{T L}{=} \frac{\omega^{2}}{2 \pi^{2} c^{3}} .
$$

Finally, taking into account the expression (14) for the average current, from (27) we obtain the expression for the right-hand side of the time equation for the medium energy density

$$
\operatorname{Sp}^{(1)} \hat{I}_{n}(x) \hat{E}_{n}^{t}(x)=\left(I_{n}^{(2)}(x), E_{n}^{t}(x)\right)+I_{n}^{(2)}(x) E_{n}^{t}(x)-\frac{2 d^{2} \omega_{0}^{4}}{3 c^{3}} n(x) \quad(\text { at } \alpha \rightarrow 0) .
$$

Note, that in fact here $\left(I_{n}^{(2)}(x), E_{n}^{t}(x)\right)$ is the correlation function of quantities $\hat{E}_{n}^{t}(x)$ and

$$
\hat{I}_{n}^{(2)}(x)=\int_{V} d x^{\prime}\left[\sigma\left(x-x^{\prime}, \varepsilon(x)\right) \hat{E}_{n}^{t}\left(x^{\prime}\right)+c \xi\left(x-x^{\prime}, \varepsilon(x)\right) \hat{Z}_{n}\left(x^{\prime}\right)\right] .
$$

The last contribution to formula (29) describes the decrease of the medium energy due to dipole radiation. The first and second terms in expression (29) look natural, but the presence of the last term is somewhat unexpected, although physically clear. 
Formulas (6), (7) and (29) give the evolution equation for the energy density of the medium. According to the remark after formula (14), with the same accuracy this evolution equation can be written with the total current $I_{n}(x)$ and the total electric field $E_{n}(x)$

$$
\partial_{t} \varepsilon(x)=\left(I_{n}(x), E_{n}(x)\right)+I_{n}(x) E_{n}(x)-\frac{2 d^{2} \omega_{0}^{4}}{3 c^{3}} n(x) \quad(\alpha \rightarrow 0) .
$$

Thus, the medium evolution is affected by average electric and magnetic fields and their binary correlations.

\section{Dynamics of the binary correlations of the electromagnetic field in nonequilibrium atom medium}

Let us formulate an equation for field correlation dynamics in the terms of correlations of magnetic field $B_{n}(x)$ and complete electric field $E_{n}(x)$. Operator Maxwell equations (5) can be written in a compact form

$$
\hat{\xi}_{1}=i \sum_{2} \mathbf{c}_{12} \hat{\xi}_{2}-4 \pi \hat{I}_{1}
$$

where

$$
\begin{gathered}
\hat{\xi}_{\mu}=\hat{\xi}_{i n}(x), \quad \hat{\xi}_{1 n}(x)=\hat{E}_{n}(x), \quad \hat{\xi}_{2 n}(x)=\hat{B}_{n}(x) ; \\
\hat{I}_{\mu}=\hat{I}_{i n}(x), \quad \hat{I}_{1 n}(x)=0, \quad \hat{I}_{2 n}(x)=\hat{I}_{n}(x) ; \\
\hat{\xi}_{s}=\hat{\xi}_{\mu_{s}}, \quad \hat{I}_{s}=\hat{I}_{\mu_{s}}, \quad \sum_{s} \ldots=\sum_{\mu_{s}} \ldots, \quad \sum_{\mu} \ldots=\sum_{i, n} \int d x \ldots
\end{gathered}
$$

(elements of matrix $\mathbf{c}_{12}$ are kernels of the type $\operatorname{rot}_{n} \delta\left(x-x^{\prime}\right)$ of an integral operator in (32)). Analogously to evolution equations for correlations $\left(\zeta_{\mu}, \zeta_{\mu^{\prime}}\right)$ of the fields $\zeta_{\mu}$, i.e. $E_{n}^{t}, B_{n}$, obtained in [6], the corresponding equations for correlations $\left(\xi_{\mu}, \xi_{\mu^{\prime}}\right)$ have the form

$$
\partial_{t}\left(\xi_{1}, \xi_{2}\right)=i \sum_{1^{\prime}} \mathbf{c}_{11^{\prime}}\left(\xi_{1^{\prime}}, \xi_{2}\right)+i \sum_{2^{\prime}} \mathbf{c}_{22^{\prime}}\left(\xi_{1}, \xi_{2^{\prime}}\right)-4 \pi\left(I_{1}, \xi_{2}\right)-4 \pi\left(\xi_{1}, I_{2}\right) .
$$

In the less compact notations these equation can be written as

$$
\begin{gathered}
\partial_{t}\left(B_{n}^{x} B_{l}^{x^{\prime}}\right)=-c \operatorname{rot}_{n}\left(E^{x} B_{l}^{x^{\prime}}\right)-c \operatorname{rot}_{l}^{\prime}\left(B_{n}^{x} E^{x^{\prime}}\right), \\
\partial_{t}\left(B_{n}^{x} E_{l}^{x^{\prime}}\right)=-c \operatorname{rot}_{n}\left(E^{x} E_{l}^{x^{\prime}}\right)+c \operatorname{rot}_{l}^{\prime}\left(B_{n}^{x} B^{x^{\prime}}\right)-4 \pi\left(B_{n}^{x} I_{l}^{x^{\prime}}\right), \\
\partial_{t}\left(E_{n}^{x} E_{l}^{x^{\prime}}\right)=c \operatorname{rot}_{n}\left(B^{x} E_{l}^{x^{\prime}}\right)+c \operatorname{rot}_{l}^{\prime}\left(E_{n}^{x} B^{x^{\prime}}\right)-4 \pi\left(I_{n}^{x} E_{l}^{x^{\prime}}\right)-4 \pi\left(E_{n}^{x} I_{l}^{x^{\prime}}\right)
\end{gathered}
$$

(hereafter for simplicity $\left(E_{n}(x), B_{l}\left(x^{\prime}\right)\right) \equiv\left(E_{n}^{x} B_{l}^{x^{\prime}}\right)$ and so on).

In order to find the material equations for evolution equations (34), (35) one has to calculate nonequilibrium correlation functions $\left(B_{n}^{x} I_{l}^{x^{\prime}}\right),\left(E_{n}^{x} I_{l}^{x^{\prime}}\right)$ and express them through the fields $\xi_{\mu}$ and their correlations $\left(\xi_{\mu}, \xi_{\mu^{\prime}}\right)$. The necessary calculations are very close to 
ones conducted in the previous section where $x^{\prime}=x$ and $l=n$. The correlations are found in the main (second) approximation in the field-medium interaction (formally speaking, in dipole moment of an atom $d$ ). Therefore, it is enough to consider the correlation function

$$
\left(E_{n}^{t x} I_{l}^{x^{\prime}}\right)=\operatorname{Sp} \rho(\eta, \varepsilon) \hat{E}_{n}^{t}(x) \hat{I}_{l}\left(x^{\prime}\right)-E_{n}^{t}(x) I_{l}\left(x^{\prime}\right) .
$$

Following the previous section, we obtain

$$
\begin{gathered}
\left(E_{n}^{t x} I_{l}^{x^{\prime}}\right)^{(1)}=0, \quad\left(E_{n}^{t x} I_{l}^{x^{\prime}}\right)^{(2)}=\left(E_{n}^{t x} I_{l}^{x^{\prime}}\right)_{1}^{(2)}+\left(E_{n}^{t x} I_{l}^{x^{\prime}}\right)_{2}^{(2)}, \\
\left(E_{n}^{t x} I_{l}^{x^{\prime}}\right)_{1}^{(2)}=-\frac{4 d^{2}}{3 \hbar^{2}} \int_{0}^{+\infty} d \omega w_{\alpha}(\omega) \frac{1}{V^{2}} \sum_{k, k^{\prime}} e^{i k x^{\prime}}\left\{\left(E_{n}^{t}(x) E_{l k}^{t}\right) \frac{\pi}{2} \delta\left(\omega-\omega_{k}\right)+\right. \\
\left.+\left(E_{n}^{t}(x) Z_{l k}\right) c \mathrm{P} \frac{1}{\omega^{2}-\omega_{k}^{2}}\right\} \varepsilon\left(x^{\prime}\right)+ \\
+i \frac{8 \pi d^{2}}{3 \hbar} \int_{0}^{+\infty} d \omega w_{\alpha}(\omega) \frac{1}{V} \sum_{k} e^{i k\left(x-x^{\prime}\right)} \varepsilon\left(x^{\prime}\right) \delta_{n l}^{t} \mathrm{P} \frac{\omega_{k}^{2}}{\omega^{2}-\omega_{k}^{2}}, \\
\left(E_{n}^{t x} I_{l}^{x^{\prime}}\right)_{2}^{(2)}=-\frac{8 \pi d^{2}}{3 \hbar} \int_{0}^{+\infty} d \omega w_{\alpha}(\omega) \frac{1}{V} \sum_{k} e^{i k\left(x-x^{\prime}\right)} \delta_{n l}^{t} \times \\
\times\left\{i \varepsilon\left(x^{\prime}\right) \omega_{k} \mathrm{P} \frac{1}{\omega^{2}-\omega_{k}^{2}}+\frac{\pi}{4} n\left(x^{\prime}\right) \hbar \omega \delta\left(\omega-\omega_{k}\right)\right\}
\end{gathered}
$$

(two contributions are chosen as in (15); $\delta_{n l}^{t} \equiv \delta_{n l}-k_{n} k_{l} / k^{2}$ ). Analogously to (27) these formulas give

$$
\begin{aligned}
&\left(E_{n}^{t x} I_{l}^{x^{\prime}}\right)^{(2)}=\frac{1}{V^{2}} \sum_{k} e^{i k x^{\prime}}\left\{\left(E_{n}^{t x} E_{l k}^{t}\right) \sigma\left(k, \varepsilon\left(x^{\prime}\right)\right)+\right. \\
&\left.+\left(E_{n}^{t x} Z_{l k}\right) c \xi\left(k, \varepsilon\left(x^{\prime}\right)\right)\right\}+S\left(x-x^{\prime}, n\left(x^{\prime}\right)\right) . \\
& S(x, n) \equiv-\frac{2 \pi d^{2}}{3} n \frac{1}{V} \sum_{k} e^{i k x} \delta_{l l}^{t} w_{\alpha}\left(\omega_{k}\right) \omega_{k}^{2} .
\end{aligned}
$$

Taking into account the expression (14) for an average current, from (38) after thermodynamic limit transition we obtain the final expression for $\left(E_{n}^{t x} I_{l}^{x^{\prime}}\right)^{(2)}$

$$
\begin{aligned}
& \left(E_{n}^{t x} I_{l}^{x^{\prime}}\right)^{(2)}=\left(E_{n}^{t x} I_{l}^{(2) x^{\prime}}\right)+S_{n l}\left(x-x^{\prime}, n\left(x^{\prime}\right)\right), \\
& S_{n l}(x, n)=-n \frac{\omega_{0}^{2} d^{2}}{12 \pi^{2}} \int d^{3} k e^{i k x} \delta_{n l}^{t} \quad(\alpha \rightarrow 0)
\end{aligned}
$$

The first term in (39) looks natural, but the presence of the last term $S_{n l}\left(x-x^{\prime}, n\left(x^{\prime}\right)\right)$ is somewhat unexpected. 
To calculate the correlation function $\left(B_{n}^{x} I_{l}^{x^{\prime}}\right)$, one needs nearly the same. Following again the previous section, we obtain

$$
\begin{gathered}
\left(B_{n}^{x} I_{l}^{x^{\prime}}\right)^{(1)}=0, \quad\left(B_{n}^{x} I_{l}^{x^{\prime}}\right)^{(2)}=\left(B_{n}^{x} I_{l}^{x^{\prime}}\right)_{1}^{(2)}+\left(B_{n}^{x} I_{l}^{x^{\prime}}\right)_{2}^{(2)}, \\
\left(B_{n}^{x} I_{l}^{x^{\prime}}\right)_{1}^{(2)}=-\frac{4 d^{2}}{3 \hbar^{2}} \int_{0}^{+\infty} d \omega w_{\alpha}(\omega) \frac{1}{V^{2}} \sum_{k, k^{\prime}} e^{i k x^{\prime}}\left\{\left(B_{n}(x) E_{l k}^{t}\right) \frac{\pi}{2} \delta\left(\omega-\omega_{k}\right)+\right. \\
\left.+\left(B_{n}(x) Z_{l k}\right) c \mathrm{P} \frac{1}{\omega^{2}-\omega_{k}^{2}}\right\} \varepsilon\left(x^{\prime}\right)- \\
-\frac{8 \pi c d^{2}}{3 \hbar} \int_{0}^{+\infty} d \omega w_{\alpha}(\omega) \frac{1}{V} \sum_{k} e^{i k\left(x-x^{\prime}\right)} \varepsilon\left(x^{\prime}\right) e_{n l s} k_{s} \frac{\pi}{2} \delta\left(\omega-\omega_{k}\right), \\
\left(B_{n}^{x} I_{l}^{x^{\prime}}\right)_{2}^{(2)}=\frac{8 \pi c d^{2}}{3 \hbar} \int_{0}^{+\infty} d \omega w_{\alpha}(\omega) \frac{1}{V} \sum_{k} e^{i k\left(x-x^{\prime}\right)} e_{n l m} k_{m} \times \\
\times\left\{i \varepsilon\left(x^{\prime}\right) \frac{\pi}{2} \delta\left(\omega-\omega_{k}\right)+\frac{1}{2} n\left(x^{\prime}\right) \mathrm{P} \frac{\omega^{2}}{\omega^{2}-\omega_{k}^{2}}\right\}
\end{gathered}
$$

(two contributions are chosen as in (15)). Analogously to (38) these formulas give

$$
\begin{aligned}
& \left(B_{n}^{x} I_{l}^{x^{\prime}}\right)^{(2)}=\frac{1}{V^{2}} \sum_{k} e^{i k x^{\prime}}\left\{\left(B_{n}^{x} E_{l k}^{t}\right) \sigma\left(k, \varepsilon\left(x^{\prime}\right)\right)+\right. \\
& \left.\left.+\left(B_{n}^{x} Z_{l k}\right) c \xi\left(k, \varepsilon\left(x^{\prime}\right)\right)\right)\right\}+T_{n l}\left(x-x^{\prime}, n\left(x^{\prime}\right)\right) . \\
& T_{n l}(x, n) \equiv n e_{n l m} \frac{4 \pi c d^{2}}{3} \frac{\partial}{\partial x_{m}} \int_{0}^{+\infty} d \omega w_{\alpha}(\omega) \frac{1}{V} \sum_{k} e^{i k x} \mathrm{P} \frac{\omega^{2}}{\omega^{2}-\omega_{k}^{2}}
\end{aligned}
$$

Taking here into account the expression (14) for the average current, after thermodynamic limit transition the final expression for $\left(B_{n}^{x} I_{l}^{x^{\prime}}\right)^{(2)}$ is obtained:

$$
\begin{aligned}
\left(B_{n}^{x} I_{l}^{x^{\prime}}\right)^{(2)} & =\left(B_{n}^{x} I_{l}^{(2) x^{\prime}}\right)+T_{n l}\left(x-x^{\prime}, n\left(x^{\prime}\right)\right), \\
T_{n l}(x, n) & \equiv n e_{n l m} \frac{c d^{2}}{6 \pi^{2}} \frac{\partial}{\partial x_{m}} \int d^{3} k e^{i k x} \mathrm{P} \frac{\omega_{0}^{2}}{\omega_{0}^{2}-\omega_{k}^{2}} \quad(\alpha \rightarrow 0) .
\end{aligned}
$$

The first term here looks natural, but the presence of the last term $T_{n l}\left(x-x^{\prime}, n\left(x^{\prime}\right)\right)$ is somewhat unexpected.

Formulas (39), (42) give material equations to evolution equations for correlations (36), (37) in agreement with [5].

\section{Conclusions}

The kinetics of electromagnetic field in the medium of two-level atoms fixed in space is constructed. The medium is described by the average of its energy density. The field subsystem is described by average electric and magnetic fields and their binary field-field correlations. The corresponding evolution equations are the Maxwell ones, equations for the correlations and the equation for energy density. Material equations to these equations express average current and nonequilibrium current-field correlations through the average fields and the binary field-field correlations. The evolution equations 
are constructed with the accuracy up to the second order in the field-medium interaction included.

In the considered approximation the obtained material equations to equations for correlations do not contain average fields. The material equations to the Maxwell equations do not contain field-field correlations. However, the mentioned material equations depend on the energy density of the medium. According to evolution equation for the energy density it is changed under influence both of the average fields and the field-field correlations. So, in the case of nonequilibrium medium, the evolution of the field and its correlations are connected.

\section{References}

1. Dicke, R.H. Coherence in spontaneous radiation processes / R.H. Dicke // Phys. Rev. - 1954. - Vol. 93. - P. 99 - 110.

2. Lyagushyn, S.F. To the problem of the Hamiltonian form for a system of twolevel atoms interacting with electromagnetic field / S.F. Lyagushyn, A.I. Sokolovsky // Visnik Dnipropetrovs'kogo Unìversitetu. Serìa Fizika, Radioelektronika. - 2015. - Vol. 23, Issue 22. - P. $54-59$.

3. Bogolyubov, N.N. Problemy dinamicheskoy teorii v statisticheskoy mekhanike / N.N. Bogolyubov. - M. - L.: Gostekhizdat, 1946. - 119 p.

4. Akhiezer, A.I. Methods of Statistical Physics / A.I. Akhiezer, S.V. Peletminsky. - Oxford: Pergamon Press, 1981. - 376 p.

5. Lyagushyn, S.F. Kinetics of system of emitters and nonequilibrium electromagnetic field / S.F. Lyagushyn, A.I. Sokolovsky // Physics of Elementary Particles and Nuclei. - 2010. - Vol. 41, No. 7. - P. 1035 - 1038.

6. Lyagushyn, S.F. Material equations in electrodynamics of medium consisting of two-level emitters / S.F. Lyagushyn, A.I. Sokolovsky, S.A. Sokolovsky // Journal of Physics and Electronics. - 2019. - Vol. 27(1). - P. 9 - 18.

\section{Appendix}

\section{Operators of physical quantities of electromagnetic field kinetics}

Here additional information is given to perform the necessary in the paper calculations.

The vector potential operator of the electromagnetic field in the standard notations of quantum electrodynamics (see, for example, [4]) is given by the formula

$$
\hat{A}_{n}(x)=c \sum_{k, \alpha}\left(\frac{2 \pi \hbar}{\omega_{k} V}\right)^{1 / 2} e_{\alpha, n}(k)\left(c_{\alpha k}+c_{\alpha,-k}^{+}\right) e^{i k x} .
$$

The circular polarization vectors $e_{\alpha n}(k)$ have simple properties

$$
e_{\alpha n}(k)^{*}=e_{\alpha n}(-k), \quad e_{\alpha n}(k) k_{n}=0, \quad \sum_{\alpha} e_{\alpha n}(k) e_{\alpha l}(k)^{*}=\delta_{n l}-k_{n} k_{l} / k^{2} .
$$

The transverse electric field operator is expressed by the formula

$$
\hat{E}_{n}^{t}(x)=-\frac{i}{\hbar}\left[\hat{H}_{\mathrm{f}}, \hat{A}_{n}(x)\right]=i \sum_{k, \alpha}\left(\frac{2 \pi \hbar \omega_{k}}{V}\right)^{1 / 2} e_{\alpha n}(k)\left(c_{\alpha k}-c_{\alpha,-k}^{+}\right) e^{i k x}
$$

with the free field Hamilton operator $\hat{H}_{\mathrm{f}}$ from (1). The magnetic field operator looks as 


$$
\hat{B}_{n}(x)=\operatorname{rot}_{n} \hat{A}(x)=i \sum_{k, \alpha}\left(\frac{2 \pi \hbar \omega_{k}}{V}\right)^{1 / 2}\left[k, e_{\alpha}(k)\right]_{n}\left(c_{\alpha k}-c_{\alpha,-k}^{+}\right) e^{i k x}
$$

In the paper the periodic boundary conditions and the following Fourier transform definition are used

$$
\mathrm{f}(x)=\frac{1}{V} \sum_{k} \mathrm{f}_{k} e^{i k x}, \quad \mathrm{f}_{k} \equiv \int_{V} d x \mathrm{f}(x) e^{-i k x} .
$$

Commutators of field operators are given by formulas

$$
\begin{aligned}
& {\left[\hat{E}_{n}^{t}(x), \hat{E}_{l}^{t}\left(x^{\prime}\right)\right]=0,\left[\hat{B}_{n}(x), \hat{E}_{l}^{t}\left(x^{\prime}\right)\right]=4 \pi i \hbar c e_{n l m} \frac{\partial \delta\left(x-x^{\prime}\right)}{\partial x_{m}},\left[\hat{B}_{n}(x), \hat{B}_{l}\left(x^{\prime}\right)\right]=0,} \\
& {\left[\hat{Z}_{n}(x), \hat{E}_{l}^{t}\left(x^{\prime}\right)\right]=4 \pi i \hbar c\left(\delta_{n l} \Delta-\frac{\partial^{2}}{\partial x_{n} \partial x_{l}}\right) \delta\left(x-x^{\prime}\right) \quad\left(\hat{Z}_{n}(x) \equiv \operatorname{rot}_{n} \hat{B}(x)\right) .}
\end{aligned}
$$

The operator of the electric dipole moment density (polarization) $\hat{P}_{n}(x)$ for the system under consideration is defined in (1). It is expressed via quasispin operators $\hat{r}_{a n}$ that satisfy commutation relations for spin $1 / 2$

$$
\left[\hat{r}_{a n}, \hat{r}_{b l}\right]=i \delta_{a b} e_{n l m} \hat{r}_{a m}
$$

(angular moment operator is $\mathrm{h} \hat{r}_{a n}$ ). In the quasispin space of a-th particle it is convenient to use a basis $\left|\sigma_{a}\right\rangle$ defined by the formulas

$$
\begin{gathered}
\hat{r}_{a z}\left|\sigma_{a}\right\rangle=\sigma_{a}\left|\sigma_{a}\right\rangle \quad\left(\sigma_{a}= \pm 1 / 2\right), \quad \hat{r}_{a}^{2}\left|\sigma_{a}\right\rangle=(1 / 2+1) 1 / 2\left|\sigma_{a}\right\rangle, \\
\left\langle\sigma_{a} \mid \sigma_{a}^{\prime}\right\rangle=\delta_{\sigma_{a}, \sigma_{a}^{\prime}} .
\end{gathered}
$$

The matrices $2\left\langle\sigma_{a}\left|\hat{r}_{a n}\right| \sigma_{a}^{\prime}\right\rangle$ coincide with the Pauli ones. It is convenient to calculate the traces of the product of several quasispin operators $\hat{r}_{a l}$ in the quasispin space in the representation of operators $\hat{r}_{a n}$ by Pauli matrices.

The material equations of the theory can be written in several forms according to the identities of the type

$$
\begin{gathered}
\mathrm{f}_{n k} \equiv \frac{1}{V} \sum_{k^{\prime}} \kappa\left(k, k^{\prime}, \varepsilon\right) E_{n k^{\prime}}, \quad \kappa\left(k, k^{\prime}, \varepsilon\right) \equiv \varepsilon_{k-k^{\prime}} \chi_{k^{\prime}} \\
\mathrm{f}_{n}(x)=\frac{1}{V} \sum_{k} e^{i k x} \kappa(k, \varepsilon(x)) E_{n k}=\int_{V} d x^{\prime} \kappa\left(x-x^{\prime}, \varepsilon(x)\right) E_{n}\left(x^{\prime}\right), \quad \kappa(k, \varepsilon) \equiv \varepsilon \chi_{k}
\end{gathered}
$$

where $\varepsilon_{k}$ and $\kappa(k, \varepsilon)$ are the Fourier transforms of functions $\varepsilon(x)$ and $\kappa(x, \varepsilon)$ defined according to (A.5). 